\title{
HUELLA DE CARBONO EN CADENAS PRODUCTIVAS \\ DE CAFÉ (Coffea arabica L.) CON DIFERENTES \\ ESTÁNDARES DE CERTIFICACIÓN EN COSTA RICA
}

\author{
MILENA A. SEGURA ${ }^{1}$ \\ HERNÁN J. ANDRADE ${ }^{2}$
}

\begin{abstract}
Recibido el 15 de agosto de 2012 y aprobado el 29 de noviembre de 2012
\end{abstract}

\section{RESUMEN}

Se estudió el impacto en la producción de café con diferentes estándares de certificación (producción convencional, producción orgánica -NOP y Unión Europea, UTZ Kapeh, Comercio Justo, Rainforest Alliance y CAFE Practices) sobre la huella de carbono en Costa Rica. Las emisiones de gases de efecto invernadero (GEI) se estimaron en nueve fincas y ocho empresas procesadoras del grano. Se estimó la fijación de carbono en biomasa total, en árboles de sombra y cafetos, midiendo las plantas, empleando modelos de biomasa y factores de expansión de biomasa, una fracción de carbono de 0,5 e indagando a productores sobre la edad de los componentes del sistema. Se emplearon factores de emisión recomendados por el IPCC (Intergovernmental Panel on Climate Change). Los sistemas de producción fijaron entre 5,0 y 17,6 t $\mathrm{CO}_{2}$ e/ha/año, sin un efecto de los estándares de certificación. La actividad que más emite GEl fue la fertilización nitrogenada (63-82\% del total de emisiones). Las dos procesadoras con menor emisión de GEI (156 y $187 \mathrm{~kg} \mathrm{CO} \mathrm{CO}_{2} \mathrm{e} / \mathrm{t}$ café verde) son aquellas que emplean la energía solar para secar parcialmente el café. La cadena de producción de café en Costa Rica mostró ser amigable con el medio ambiente, al fijar netamente entre 2,4 y 13,0 $\mathrm{kg} \mathrm{CO} \mathrm{CO}_{2} \mathrm{e} \mathrm{kg}$ de grano de café verde (en promedio $7,6 \mathrm{~kg}$ $\mathrm{CO}_{2} \mathrm{e} / \mathrm{kg}$ de café verde y 8,1 t $\mathrm{CO}_{2} \mathrm{e} / \mathrm{ha} / \mathrm{año}$ ). No se encontró impacto de los estándares de certificación pero si de los componentes del sistema en la huella de carbono en la cadena de producción de café.

\section{PALABRAS CLAVE:}

Biomasa, combustibles fósiles, fertilización nitrogenada, mitigación, procesamiento. 


\title{
CARBON FOOTPRINTS IN THE COFFEE (Coffea arabicaL.) PRODUCTIVE CHAINS WITH DIFFERENT CERTIFICATION STANDARDS IN COSTA RICA
}

\begin{abstract}
The impact in coffee production with different certification standards (conventional production, organic production NOP and European Union-, UTZ Kapeh, Fairtrade, Rainforest Alliance and CAFE Practices) on Carbon Footprint in Costa Rica was studied. The greenhouse gas emissions (GHG) were estimated in nine farms and eight grain processing industries. Carbon fixation was estimated in total biomass in both shade trees and coffee bushes by measuring the plants, using biomass models and biomass expansion factors, a 0.5 fraction of carbon and asking producers about the age of the components in the system. IPCC (Intergovernmental Panel on Climate Change) recommended emission factors were used. The production systems fixed between 5,0 and 17,6 t $\mathrm{CO}_{2} \mathrm{e} /$ ha/year, without an effect of the certification standards. The activity emitting more GHG was nitrogenated fertilization $(63-82 \%$ of total emissions). The two grain processing industries with less GEE emissions (156 and $187 \quad \mathrm{CO}_{2} \mathrm{e} / \mathrm{t}$ green coffee) are those using solar energy for partial coffee drying. The coffee production chain in Costa Rica showed to be friendly with the environment while fixing a net between 2.4 and $13.0 \mathrm{~kg} \mathrm{CO} \mathrm{CO}_{2} \mathrm{e} / \mathrm{kg}$ of green coffee grain $\left(7.6 \mathrm{~kg} \mathrm{CO} \mathrm{CO}_{2} \mathrm{e} / \mathrm{kg}\right.$ of green coffee average and $8.1 \mathrm{t} \mathrm{CO}_{2} \mathrm{e} /$ ha/year). Impact on the certification standards was not found but it was found in the system components of the carbon footprint in the coffee production chain.
\end{abstract}

\section{KEY WORDS:}

Biomass, fossil fuel, nitrogenated fertilization, mitigation, processing

\section{INTRODUCCIÓN}

El aumento en la concentración de gases de efecto invernadero (GEI), desde la era industrial, ha acrecentado el cambio climático global (IPCC, 2003). Los GEI más importantes son el vapor de agua, dióxido de carbono $\left(\mathrm{CO}_{2}\right)$, ozono $\left(\mathrm{O}_{3}\right)$, metano $\left(\mathrm{CH}_{4}\right)$ y óxido nitroso $\left(\mathrm{N}_{2} \mathrm{O}\right)$, siendo los últimos cuatro los más afectados por las actividades antropogénicas. Las actividades agrícolas contribuyen con el $13,5 \%$ de las emisiones mundiales de GEI (IPPC, 2007), producto del $\mathrm{CH}_{4}$ y $\mathrm{N}_{2} \mathrm{O}$ (Johnson; 
Fransluebbers; Weyers \& Reicosky, 2007), provenientes de la fermentación entérica, producción bajo inundación y fertilización nitrogenada. Sin embargo, los sistemas forestales y agroforestales pueden absorber cantidades significativas de carbono en la biomasa y el suelo (Albrecht \& Kandji, 2003; Amézquita; Ibrahim; Llanderal; Buurman \& Amézquita, 2005; Andrade; Brook \& Ibrahim, 2008; Beer, et al. 2003; Montagnini \& Nair, 2004; Oelbermann; Voroney \& Gordon, 2004; Soto-Pinto, Anzueto; Mendoza, JiménezFerrer \& De Jong, 2010) y mitigar el problema del cambio climático. Dicho servicio ambiental generado por estos ecosistemas puede ser sujeto de pago por servicios ambientales.

Los pagos por servicios ambientales incluyen el esquema basado en productos, en los cuales los consumidores pagan una prima "verde" sobre el producto que está certificado como amigable con el medio ambiente. Es decir, los consumidores finales del producto están pagando por un beneficio ambiental de tales sistemas de producción. En este esquema, se puede incluir la producción de café orgánico con sombrío (Wunder, 2007). En el Mundo, existen alrededor de 25 millones de productores de café (Giovannucci \& Koekoek, 2003); no obstante, la producción de café certificado, tal como el Comercio Justo, orgánico y ecológico, es aún pequeña (menos del $2 \%$ ) en los mercados más desarrollados (Japón y mayoría de mercados europeos), pero incluyen beneficios adicionales a los 750 mil hogares y las industrias en toda la cadena de producción (Giovannucci \& Koekoek, 2003). Los actuales programas de certificación de café se dividen en tres tipos, los cuales no son mutuamente excluyentes: orgánicos, Comercio Justo y de sombrío. Tales esquemas de certificación promueven la protección de la biodiversidad y podrían mejorar los medios de vida de los agricultores (Philpott; Bichier; Rice \& Greenberg, 2007).

Los estándares de certificación no incluyen indicadores específicos sobre la huella de carbono a nivel de finca; aunque, todos los estándares evaluados (UTZ Kapeh, Comercio Justo, Producción Orgánica -NOP y Unión Europea-, Rainforest Alliance y CAFE Practices) promueven la conservación ambiental, tal como la fertilización orgánica, la prevención de la erosión y el uso eficiente de maquinaria (Cuadro 1). Los estándares de Comercio Justo, UTZ Kapeh, Producción Orgánica y CAFE Practices incluyen el mantenimiento o mejoramiento de la cobertura arbórea a nivel de finca y plantación de café. La certificación de Rainforest requiere de más de un $40 \%$ de sombra de al menos 70 individuos por hectárea, de un mínimo de 12 especies nativas, con dos o más estratos. La huella de carbono en la etapa de procesamiento podría ser 
afectada por los requerimientos de los estándares de Comercio Justo, UTZ Kapeh y CAFE Practices, las cuales promueven el uso de energía renovable incluyendo la radiación solar y la quema de leña proveniente de la poda de árboles de sombra.

Este estudio desarrolló una metodología para estimar el impacto de los sistemas y estándares de certificación de la producción de café en Costa Rica en la huella de carbono. La huella de carbono en este estudio incluye el secuestro de carbono en biomasa total y las emisiones de GEI a nivel de finca y procedentes del procesamiento para obtener café oro. Este estudio es de gran relevancia porque además se enmarca en la política nacional de Costa Rica de alcanzar la neutralidad de carbono para el 2021.

\section{MATERIALES Y MÉTODOS}

La huella de carbono de la cadena productiva de café en Costa Rica fue calculada con base en mediciones de árboles de sombra y arbustos de café en pie y en estimados de emisiones GEI en las actividades de manejo en los cafetales y del procesamiento del grano. La huella de carbono se basó en sistemas de producción de café ya establecidos, por lo cual sólo se consideraron las emisiones de GEI generadas en el manejo del sistema de producción.

\begin{tabular}{|c|c|c|c|}
\hline $\begin{array}{l}\text { Esquema de } \\
\text { certificación }\end{array}$ & Uso de energía & $\begin{array}{c}\text { Uso de } \\
\text { fertilizantes } \\
\text { nitrogenados }\end{array}$ & $\begin{array}{c}\text { Manejo de vegetación en } \\
\text { cafetales }\end{array}$ \\
\hline UTZ Kapeh & $\begin{array}{lr}\text { Prácticas } & \text { para } \\
\text { minimizar erosion. Uso } \\
\text { eficiente de energía, } \\
\text { energía solar si es } \\
\text { posible y leña de } \\
\text { podas. }\end{array}$ & $\begin{array}{l}\text { Uso de especies } \\
\text { fijadoras } \\
\text { nitrógeno }\end{array}$ & $\begin{array}{l}\text { Uso de árboles de sombra y } \\
\text { cultivos de cobertura, } \\
\text { preferiblemente nativos. } \\
\text { Deforestación es prohibida }\end{array}$ \\
\hline Comercio Justo & $\begin{array}{lr}\text { Minimizar. } & \text { Usar } \\
\text { principalmente } & \text { no } \\
\text { renovable } & \\
\end{array}$ & Uso regulado & $\begin{array}{l}\text { Promoción de reforestación y } \\
\text { uso de cobertura arbórea. } \\
\text { Deforestación es prohibida }\end{array}$ \\
\hline NOP orgánico & $\begin{array}{l}\text { Prácticas que } \\
\text { minimicen erosión }\end{array}$ & $\begin{array}{l}\text { Uso de materiales } \\
\text { orgánicos } \\
\text { (compostados y sin } \\
\text { compostar) }\end{array}$ & $\begin{array}{l}\text { Rotaciones, cultivos de } \\
\text { cobertura }\end{array}$ \\
\hline Unión Europea & $\begin{array}{l}\text { Uso responsable de } \\
\text { energía }\end{array}$ & $\begin{array}{l}\text { Uso de abonos } \\
\text { verdes, fertilizadión } \\
\text { orgánica, } \\
\text { preferiblemente } \\
\text { compostado }\end{array}$ & $\begin{array}{l}\text { Uso de leguminosas } 0 \\
\text { plantas de raíz profunda, } \\
\text { rotación de cultivos }\end{array}$ \\
\hline Rainforest & $\begin{array}{l}\text { Prácticas de prevendion } \\
\text { de erosión }\end{array}$ & $\begin{array}{l}\text { Se recomienda el } \\
\text { uso de coberturas } \\
\text { y rotación de } \\
\text { cultivos }\end{array}$ & $\begin{array}{l}\text { Arboles de sombra }(>70 \\
\text { árboles/ha de }>12 \text { especies } \\
\text { nativas/ha y }>40 \% \text { de } \\
\text { sombra permanente con al } \\
\text { menos dos estratos) }\end{array}$ \\
\hline CAFE Practices & $\begin{array}{l}\text { Minimizar. Uso de } \\
\text { energía renovable, } \\
\text { como solar y otras más } \\
\text { eficientes }\end{array}$ & No especifica & $\begin{array}{l}\text { Sombra imitando las } \\
\text { condiciones naturales } \\
\text { cuando sea posible. } \\
\text { Mantenercapa de hojarasca }\end{array}$ \\
\hline
\end{tabular}


Fuente: http://www.utzcertified-trainingcenter.com, http://www.fairtrade.net/fileadmin/user_upload/content/20 09/standards/documents/2012-04-01_SP_Coffee_SPO.pdf, http://www.ecfr.gov/cgi-bin/text-

idx?c=ecfr\&sid=3f34f4c22f9aa8e6d 9864cc2683cea02\&tpl=I ecfrbrowse/Title07/7cfr205_main_02.tpl, http:/lec.europa.eulagriculture/organic/organicfarming/what-organic/the-farm_es, http://sanstandards.org/userfiles/SAN-S-11\%20SAN\%20Sustainable \%20Agriculture\%20Standard\%20July\%202010\%20v2.pdf, http://www.scscertified.com/retail/docs/ CAFEPracticesEvaluationGuidelines010307_esp.pdf.

La cadena de producción de café se dividió en dos actividades principales: a nivel de finca y el procesamiento. Este estudio se desarrolló mediante las siguientes etapas metodológicas:

\section{SELECCIÓN DE SISTEMAS DE PRODUCCIÓN Y FINCAS}

Se seleccionaron nueve fincas cafeteras en Costa Rica, dos de cada uno de los siguientes esquemas de certificación: producción convencional (sin certificación), producción orgánica (NOP y Unión Europea) y UTZ Kapeh $y$; una con certificación de Comercio Justo, Rainforest Alliance y CAFE Practices. Estas fincas fueron seleccionadas por el Proyecto COSA (por sus siglas en inglés Committee on Sustainability Assessment) y estaban distribuidas en las principales zonas cafeteras del país: Plan de Birrí, San Rafael de Heredia, La Suiza, Aquiares y Colorado de Turrialba, San Marcos de Tarrazú, Llano Bonito (zona de Los Santos), Grecia y Naranjo. Las fincas seleccionadas tenían café en sistemas agroforestales (SAF) con una o varias de las siguientes especies arbóreas: Inga spp, Cordia alliodora, árboles frutales, Eucalyptus spp, Pinus spp y Cupressus Iusitanica (Cuadro 2). 


\begin{tabular}{|c|c|c|c|}
\hline Sitio & Altitud $(\mathrm{m})$ & $\begin{array}{l}\text { Especies arbóreas de } \\
\text { sombra }\end{array}$ & $\begin{array}{l}\text { Esquema de } \\
\text { certificación }\end{array}$ \\
\hline Plan de Birrí & 1600 & Cupressus lusitanica & UTZ Kapeh \\
\hline San Rafael de Heredia & 1300 & Cupressus Iusitanica & UTZ Kapeh \\
\hline Llano Bonito & 1400 & Frutales y Pinus spp & Comercio Justo \\
\hline Colorado de Turrialba & 930 & $\begin{array}{l}\text { Cordia alliodora, Inga } \\
\text { spp, Eucalyptus spp y } \\
\text { frutales }\end{array}$ & $\begin{array}{l}\text { Orgánico (NOP y } \\
\text { Unión Europea) }\end{array}$ \\
\hline Grecia & $800-900$ & $\begin{array}{l}\text { Eucalyptus spp y } \\
\text { Cordia alliodora }\end{array}$ & $\begin{array}{l}\text { Orgánico (NOP y } \\
\text { Unión Europea) }\end{array}$ \\
\hline Naranjo & $1350-1400$ & $\begin{array}{l}\text { Eucalyptus spp y } \\
\text { Cordia alliodora }\end{array}$ & Rainforest \\
\hline Aquiares de Turrialba & $820-1400$ & $\begin{array}{l}\text { Eucalyptus spp y } \\
\text { Cordia alliodora }\end{array}$ & CAFE Practices \\
\hline La Suiza de Turrialba & 600 & Cordia alliodora & $\begin{array}{l}\text { Producción } \\
\text { convencional }\end{array}$ \\
\hline San Marcos de Tarrazú & 1500 & $\begin{array}{l}\text { Eucalyptus spp y } \\
\text { Cupressus lusitanica }\end{array}$ & $\begin{array}{l}\text { Producción } \\
\text { convencional }\end{array}$ \\
\hline
\end{tabular}

\section{Estimación del almacenamiento de carbono y las tasas de fijación en los sistemas de producción}

La biomasa arriba del suelo se estimó calculando la densidad de plantación y la biomasa promedio por individuo por componente (cafetos y árboles de sombra). La densidad de plantación fue estimada en parcelas temporales de muestreo rectangulares de $500 \mathrm{~m}^{2}$ para árboles y $30 \mathrm{~m}^{2}$ para cafetos.

La biomasa promedio por individuo se estimó mediante la medición de la altura total (ht) y el diámetro a la altura del pecho (dap) de 10 árboles con dap $\geq 10 \mathrm{~cm}$ y la ht y el diámetro del tronco a $15 \mathrm{~cm}$ de altura $\left(D_{15}\right)$ de 10 cafetos seleccionados aleatoriamente en todo el sistema estudiado. Las dimensiones de las leñosas perennes fueron transformadas a biomasa arriba del suelo empleando modelos de biomasa reportados en la literatura (Cuadro 3). La mayoría de estas ecuaciones se han desarrollado en sistemas agroforestales con café y cacao en Nicaragua y Costa Rica, respectivamente. La biomasa aérea de Eucalyptus spp y Cupressus lusitanica se calculó con una ecuación de volumen de madera (Cuadro 3), el peso específico de la madera de 0,4 y $0,5 \mathrm{~g} / \mathrm{cm}^{3}$ y el factor de expansión de la biomasa para el volumen total de 1,2 y 1,4, respectivamente. La biomasa de los árboles podados, como Erythrina spp, se calculó midiendo la altura total y el diámetro en el centro del tronco principal y una gravedad específica de $0,25 \mathrm{~g} / \mathrm{cm}^{3}$ (Ávila, 2000). El almacenamiento de carbono en la biomasa total se calculó utilizando el valor default de fracción de carbono (0,5; IPCC, 2003).

La biomasa bajo el suelo se estimó a través de una ecuación general desarrollada por Cairns; Brown; Helmer \& Baumgardner (1997) y recomendada por el IPCC (2003): 


$$
B r=e^{\left(-1,0587+0,8836^{*} \operatorname{Ln}(B a)\right)}
$$

Donde;

$\mathrm{Br}$ : Biomasa bajo el suelo (t/ha)

Ba: Biomasa arriba del suelo (t/ha)

Cuadro 3. Modelos alométricos para estimar la biomasa arriba del suelo de los árboles y los arbustos de café que crecen en los sistemas agroforestales.

\begin{tabular}{|c|c|c|c|}
\hline Especies & Modelo & $\mathrm{R}^{2}$ & Fuente \\
\hline Inga spp & $B=10^{\left(-0,889+2,317^{*} \log (d a p)\right)}$ & 0,96 & \multirow{3}{*}{$\begin{array}{l}\text { Segura, } \\
\text { Kanninen \& } \\
\text { Suárez, } \\
2006\end{array}$} \\
\hline Cordia alliodora & $B=10^{\left(-0,755+2,072^{*} \log (d a p)\right)}$ & 0,95 & \\
\hline Coffea arabica & $B=10^{\left(-1,113+1,578^{*} \log \left(D_{15}\right)+0,581^{*} \log (h t)\right)}$ & 0,94 & \\
\hline $\begin{array}{l}\text { Arboles frutales } \\
\text { (modelo } \\
\text { multiespecies) }\end{array}$ & $B=10^{\left(-1,12+2,62 * \log (d a p)+0,03^{*} \log (h t)\right)}$ & 0,95 & $\begin{array}{l}\text { Andrade, } \\
\text { Segura \& } \\
\text { Somarriba, } \\
\text { en } \\
\text { preparación }\end{array}$ \\
\hline Eucalyptus spp & $V=e^{\left(-10,54+1,99^{*} \operatorname{Ln}(d a p)+1,08^{*} \operatorname{Ln}(h t)\right)}$ & 0,99 & $\begin{array}{l}\text { Centro } \\
\text { Agronómico } \\
\text { Tropical de } \\
\text { Investigación } \\
\text { y Enseñanza } \\
\text { (CATIE), } \\
1994 \\
\end{array}$ \\
\hline Pinus spp & $B=0,89+\left(\frac{10486^{*} d a p^{2,84}}{d a p^{2,84}+376907}\right)$ & 0,98 & IPCC, 2005 \\
\hline $\begin{array}{l}\text { Cupressus } \\
\text { lusitanica }\end{array}$ & $V=-0,013+2,6 \times 10^{-5} * d a p^{2} * h t$ & -- & $\begin{array}{l}\text { Chaves \& } \\
\text { Fonseca, } \\
1991\end{array}$ \\
\hline
\end{tabular}

B: biomasa arriba del suelo ( $\mathrm{kg} / \mathrm{planta})$; dap: diámetro del tallo del árbol a la altura del pecho $(\mathrm{cm}) ; \mathrm{D}_{15}$ : diámetro del tronco a $15 \mathrm{~cm}$ de altura $(\mathrm{cm})$; ht: altura total $(\mathrm{m})$. V: volumen total del tronco $\left(\mathrm{m}^{3}\right)$.

La tasa de fijación de carbono en cada componente (árboles y arbustos de café) fue estimada dividiendo el almacenamiento de carbono en la biomasa total entre la edad promedio de cada componente. La edad de cada componente fue estimada mediante la realización de una entrevista al productor o administrador de la finca. La relación entre el almacenamiento y la fijación de carbono se utilizó para estimar la tasa de fijación de carbono a escala del sistema. En el caso de edad desconocida, la tasa de fijación de carbono se calculó considerando las curvas de crecimiento de cada especie, además de una relación entre el almacenamiento de carbono y la tasa de fijación.

\section{Estimación de las emisiones de GEI en el manejo de fincas cafeteras y en procesamiento de café}


Nueve fincas cafeteras y ocho industrias procesadoras de café en Costa Rica fueron seleccionadas y entrevistadas en el 2008. Los procesadores de grano que reciben las cerezas de café de las fincas estudiadas fueron entrevistados: Asociación de Productores Orgánicos de Turrialba (APOT), Beneficio La Eva, Beneficio Santa Rosa (Cafetalera Orlich), la Asociación Cafetalera Aquiares SA, Coopedota RL (Cooperativa de Caficultores de Dota RL), Coopelibertad RL (Cooperativa de Caficultores de Heredia Libertad RL), Coopellanobonito RL (Cooperativa de Caficultores de Llano Bonito RL) y Coopronaranjo RL (Cooperativa de Productores de Café y Servicios Múltiples de Naranjo RL).

Se realizaron entrevistas semiestructuradas para hacer una reconstrucción de las actividades de manejo de cada sistema de producción de café. Se formularon las preguntas para estimar el uso de cal, energía, combustibles fósiles y fertilizantes nitrogenados. En el caso de encontrarse vacíos de información o contradicciones en las respuestas de los productores, se procedió a consultar a expertos en el manejo de plantaciones de café en las zonas de estudio.

Los resultados de los flujos de GEl se calcularon en términos de un año de producción (proceso) y por hectárea en el caso de las fincas. Al final, todos los valores se expresan como los flujos de GEI por cada unidad de grano de café verde producido. Las emisiones producto del procesamiento de café fueron estimadas mediante encuestas y/o consultando los registros de la industria, por ejemplo, las facturas del servicio de electricidad. Las siguientes son las actividades que emiten gases de efecto invernadero a la atmósfera en los procesos de manejo de las plantaciones y procesamiento del grano de café:

a. Aplicación de fertilizantes nitrogenados y carbonatos. La cantidad de nitrógeno y carbonatos aplicada anualmente a las plantaciones de café fue estimada mediante una encuesta, en la cual se incluía el tipo y dosis de fertilizante y cal. Se consideró un factor de emisión de la aplicación de nitrógeno de $0,01 \mathrm{~kg} \mathrm{~N} \mathrm{~N}_{2} \mathrm{O} / \mathrm{kg} \mathrm{N}$ (IPCC, 2006). La aplicación de los carbonatos genera una emisión de 0,12 y 0,122 $\mathrm{kg} \mathrm{C} / \mathrm{kg}$ de carbonato de calcio y magnesio, respectivamente (IPCC, 2006).

b. Uso de combustibles fósiles. Los combustibles fósiles utilizados en el manejo de las plantaciones de café (renovación de las plantaciones y aplicaciones de agroquímicos), el transporte de granos de café, personal, leña y otros insumos, fueron estimados por medio de entrevistas. Se preguntó por la cantidad de combustibles 
fósiles utilizados en todas las etapas de la cadena de producción del café. Si tal información no estaba disponible, el volumen de éstos se estimó mediante el tiempo de trabajo o la distancia recorrida y la eficiencia del uso de combustibles fósiles. Las estimaciones de uso de combustibles fósiles se hicieron sólo para la aplicación de los insumos. Los factores de emisión utilizados fueron de 2,83 y $2,33 \mathrm{~kg} \mathrm{CO}_{2} \mathrm{e} / \mathrm{l}$ de diesel y gasolina, respectivamente (IPCC, 2006).

c. Uso de leña u otros combustibles. El consumo de leña fue estimado en las entrevistas y/o revisando los registros de los beneficiaderos. La leña usada en el secado del grano de café emite $\mathrm{GEI}$, a razón de 957,25 $\mathrm{g} \mathrm{CO}_{2} \mathrm{e} / \mathrm{kg}$ leña (950 g de $\mathrm{CO}_{2} ; 0,05 \mathrm{~g} \mathrm{CH}_{4}$ y $0,02 \mathrm{~g}$ de $\mathrm{N}_{2} \mathrm{O} / \mathrm{kg}$ leña; $\mathrm{EC}$, 2007). El uso de leña se registró en $\mathrm{m}^{3}$ estéreos, unidad de medida tradicional para este país, los cuales se convirtieron a volumen sólido utilizando un factor de $78 \%$ (González, 2001) y en peso seco suponiendo una densidad de la madera de $0,4 \mathrm{~g} / \mathrm{cm}^{3}$. También se consideró un factor de oxidación del $87 \%$ (IPCC, 2006).

d. Uso de electricidad. La emisión de GEI debido a la electricidad se estimó con base en la cantidad de ésta en el procesamiento del grano y el factor de emisión. El primer parámetro fue estimado a través de la entrevista y el consumo de electricidad reportado en el último recibo eléctrico del año. Se empleó un factor de emisión de 6,6 kg $\mathrm{CO}_{2} / \mathrm{MWh}$, el cual se estimó con base en: 1) el método de generación de electricidad de Costa Rica, de acuerdo a las estadísticas nacionales (MIDEPLAN, 2008), y 2) el factor de emisión de acuerdo al tipo de insumo empleado para la generación (0,60 t $\mathrm{CO}_{2} / \mathrm{MWh}$ para diesel y 0 t $\mathrm{CO}_{2} / \mathrm{MWh}$ para hidroeléctricas, geotérmicas y eólicas; World Resource Institute (WRI) \& World Business Council for Sustainable Development (WBCSD), 2007).

\section{Construcción de la huella de carbono de la cadena de suministro de café}

La huella de carbono siguió el esquema en el proceso de producción y transformación del café (Figura 1). Con base en las tasas de fijación de carbono en biomasa total y las emisiones a nivel de finca y beneficiadero, se procedió a estimar la huella de carbono por unidad de café oro procesado. A cada productor de café seleccionado se le indagó acerca de la producción promedio de café en cereza en el sistema de producción evaluado. La capacidad de calentamiento del $\mathrm{CH}_{4}$ y $\mathrm{N}_{2} \mathrm{O}$ fue usada para estimar las emisiones en términos de $\mathrm{CO}_{2} \mathrm{e}: 21 \mathrm{~g} \mathrm{CO}_{2} \mathrm{e} / \mathrm{g} \mathrm{CH}_{4}$ y $310 \mathrm{~g}$ $\mathrm{CO}_{2} \mathrm{e} / \mathrm{g} \mathrm{N}_{2} \mathrm{O}$ (IPCC, 2006). 


\section{RESULTADOS Y DISCUSIÓN}

\section{Almacenamiento y fijación de carbono en la biomasa total}

La tasa de fijación de carbono en la biomasa total varió entre 5,0 y 17,6 t $\mathrm{CO}_{2}$ e/ha/año en los sistemas de producción de café evaluados (Cuadro 4). Alrededor del $87 \%$ de la fijación de carbono fue acumulada por los árboles de sombra (incluyendo maderables, de servicio y frutales), y el restante en los arbustos de café (8,7 vs 1,2 $\mathrm{CO}_{2} \mathrm{e} / \mathrm{ha} / \mathrm{año}$, respectivamente; Cuadro 4).

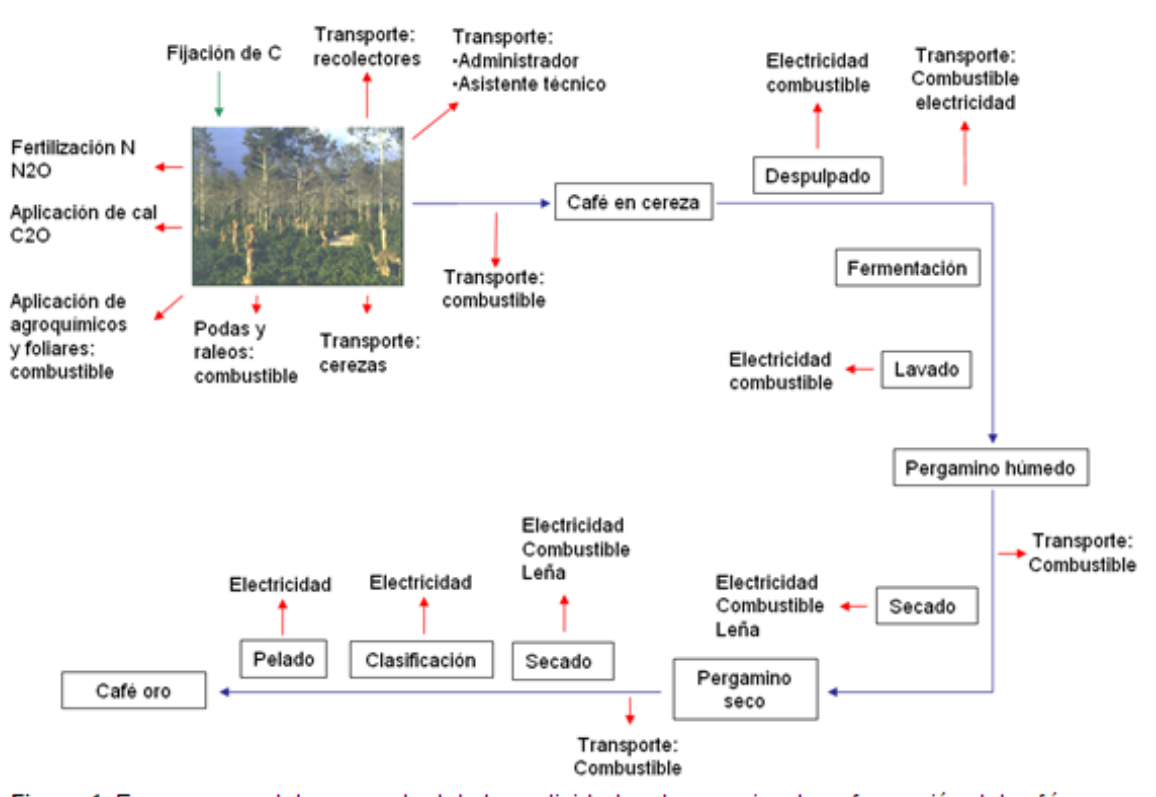

Figura 1. Esquema-modelo conceptual de las actividades de manejo y transformación del café que afectan la huella de carbono.

Las flechas rojas indican actividades que emiten gases de efecto invernadero. La flecha verde señala la actividad que fija carbono atmosférico en biomasa.

Cuadro 4. Tasas de fijación de carbono en la biomasa total de las plantaciones de café bajo diferentes estándares de certificación en Costa Rica.

\begin{tabular}{|c|c|c|c|c|c|}
\hline \multirow{3}{*}{$\begin{array}{l}\text { Estándar de } \\
\text { certificación }\end{array}$} & \multirow{3}{*}{$\begin{array}{l}\text { Producción } \\
{ }^{\star}(\mathrm{kg} / \mathrm{ha} / \mathrm{año})\end{array}$} & \multicolumn{4}{|c|}{ Tasa de fijación } \\
\hline & & \multicolumn{3}{|c|}{ (t CO 2 e/ha/año) } & $\left(\mathrm{kg} \mathrm{CO}_{2} \mathrm{e} / \mathrm{kg}^{*}\right)$ \\
\hline & & Arboles & Cafetos & Total & Total \\
\hline Rainforest & 1583 & 4,2 & 1,6 & 5,9 & 3,7 \\
\hline Comercio Justo & 1840 & 11,6 & 0,2 & 11,8 & 6,4 \\
\hline UTZ Kapeh & 1204 & 6,8 & 1,1 & 8,0 & 6,7 \\
\hline $\begin{array}{l}\text { Producción } \\
\text { convencional }\end{array}$ & 1116 & 11,8 & 2,1 & 13,9 & 14,0 \\
\hline $\begin{array}{l}\text { Producción } \\
\text { Orgánica }\end{array}$ & 463 & 9,1 & 0,8 & 9,8 & 35,0 \\
\hline Promedio & 1241 & 8,7 & 1,2 & 9,9 & 13,2 \\
\hline
\end{tabular}

Los sistemas convencionales de producción de café fijaron más carbono que las plantaciones bajo esquemas de 
Comercio Justo, Orgánico, UTZ Kapeh y Rainforest Alliance $\left(13,9 ; 11,8 ; 9,8 ; 8,0\right.$ y 5,9 t $\mathrm{CO}_{2} \mathrm{e} / \mathrm{ha} / \mathrm{año}$, respectivamente; Cuadro 4). Se encontraron diferencias superiores en el secuestro de carbono por unidad de café de grano verde producido: la producción orgánica fijó una considerablemente mayor cantidad de carbono atmosférico (35,0 $\mathrm{kg} \mathrm{CO}_{2} \mathrm{e} / \mathrm{kg}$ grano de café verde) que los otros sistemas (3,7 a 14,0 kg CO 2 e/kg café verde; Cuadro 4).

Las características de los árboles de sombra, tal como la densidad y composición botánica, el tamaño de los individuos, la tasa crecimiento y la edad de la madera, tienen una mayor influencia sobre las tasas de fijación de carbono que los sistemas certificados. Estudios de fijación de carbono en biomasa en SAF con café han arrojado tasas de entre 7,7 y 16,9 t $\mathrm{CO}_{2} \mathrm{e} /$ ha/año (Ávila; Jiménez; Beer; Gómez \& Ibrahim, 2001; Aristizábal y Guerra 2002; Hergoualc'h; Blanchart; Skiba; Henault \& Harmand, 2012; Andrade; Marín; Pachón \& Segura, en edición), dentro de las cuales se encuentran las estimaciones de este estudio. Se ha demostrado que los SAF con café fijan más carbono atmosférico en la biomasa que aquellos en monocultivo (Oelbermann, et al. 2004; Avilés, 2009).

\section{Emisiones de GEI en las fincas cafeteras}

No hay diferencias aparentes en las emisiones de GEI entre los estándares de certificación. Casi todas las fincas de café emitieron entre 0,5 y 1,1 $\mathrm{kg} \mathrm{CO} / \mathrm{kg}$ de café verde de grano producido (Cuadro 5). Sin embargo, se encontró una finca orgánica atípica, cuyo factor de emisión es increíblemente mayor que el resto de las fincas evaluadas (44,3 $\mathrm{kg} \mathrm{CO}_{2}{ }^{\mathrm{e}} / \mathrm{kg}$ del grano de café verde; Cuadro 5). Este valor puede explicarse por las altas dosis de fertilizantes orgánicos aplicados (102 t/ha/año) y el gran uso de combustibles fósiles para su transporte a la plantación de café.

La fuente de GEI más importante en las plantaciones de café fue la aplicación de nitrógeno $(63-82 \%$ de las emisiones de $\mathrm{GEI}$ ), variando entre 0,21 y $0,73 \mathrm{~kg} \mathrm{CO} \mathrm{CO}_{2} \mathrm{e} \mathrm{kg}$ de grano de café verde (excluyendo la finca orgánica, la cual presentó valores extremos), seguido por el uso de combustibles fósiles (aplicaciones de insumos y transporte), y la aplicación de los carbonatos (15-36\%; Cuadro 5). Estos resultados coinciden parcialmente con los reportes de Noponen, et al. (2012), quienes encontraron que el nitrógeno orgánico e inorgánico contribuyó con 7$42 \%$ de la huella de carbono en cafetales de Costa Rica y Nicaragua. Aunque algunos esquemas de certificación implican la reducción o eliminación de productos sintéticos, los fertilizantes nitrogenados son un factor clave en el 
crecimiento pues debe ser suplementado a las plantas. Las fincas orgánicas también aplican nitrógeno pero usando una gran cantidad de productos con un menor contenido de este nutriente.

Cuadro 5. Emisiones de gases de efecto en el invernadero debido al manejo de plantaciones de café bajo diferentes esquemas de certificación en Costa Rica.

\begin{tabular}{|c|c|c|c|c|c|c|}
\hline \multirow{2}{*}{$\begin{array}{l}\text { Estándar de } \\
\text { certificación }\end{array}$} & $\begin{array}{l}\text { Fertilizantes } \\
\text { nitrogenados }\end{array}$ & Carbonatos & Diesel & Gasolina & Total & \multirow{2}{*}{$\begin{array}{c}\text { Total } \\
\left(\mathrm{tCO}_{2} \mathrm{e} / \mathrm{ha} / \mathrm{anno}\right)\end{array}$} \\
\hline & \multicolumn{5}{|c|}{ (kg CO $\mathrm{CO}_{2} \mathrm{e} / \mathrm{kg}$ café verde) } & \\
\hline \multirow{3}{*}{$\begin{array}{l}\text { Producción } \\
\text { convencional } \\
\text { UTZ Kapeh } \\
\text { Rainforest } \\
\text { Comercio } \\
\text { Justo }\end{array}$} & 0,47 & 0,10 & 0,09 & 0,00 & 0,66 & 0,73 \\
\hline & $\begin{array}{l}0,52 \\
0,57\end{array}$ & $\begin{array}{l}0,12 \\
0,16\end{array}$ & $\begin{array}{l}0,03 \\
0,08\end{array}$ & $\begin{array}{l}0,04 \\
0,03\end{array}$ & $\begin{array}{l}0,70 \\
0,84\end{array}$ & $\begin{array}{l}0,85 \\
1,33\end{array}$ \\
\hline & 0,52 & 0,24 & 0,06 & 0,00 & 0,82 & 1,51 \\
\hline $\begin{array}{l}\text { Producción } \\
\text { orgánica }\end{array}$ & 18,68 & 0,10 & 1,78 & 2,10 & 22,66 & 10,49 \\
\hline
\end{tabular}

\section{Emisiones de GEI en el procesamiento del grano de café}

La emisión de GEl está fuertemente afectada por el tipo de energía que se emplea en el procesamiento del grano. Se encontraron diferencias notables en las emisiones de GEI entre las plantas de procesamiento. Sin embargo, dichas diferencias no pueden atribuirse al estándar de certificación debido a que la mayoría de ellas procesan el café proveniente de diferentes esquemas (Cuadro 6). Además, el café de fincas certificadas recibe el mismo proceso para obtener granos de café verde. Coopedota y Coopellanobonito presentaron las menores emisiones de GEl (156 y 187 kg CO 2 e/t café verde, respectivamente; Cuadro 6), debido a que utilizan energía solar para secar parcialmente el café. En contraste, Cafetalera Aquiares emitió la mayor cantidad de GEI por unidad de café verde producido (654 kg CO $2 \mathrm{e} / \mathrm{t}$ ); aunque, utiliza una gran cantidad de leña de la misma finca (Cuadro 6).

Cuadro 6. Emisiones de GEI de acuerdo con el uso de la energía en el procesamiento de grano de café en Costa Rica.

\begin{tabular}{|c|c|c|c|c|c|}
\hline \multirow[b]{2}{*}{ Procesador } & \multirow{2}{*}{$\begin{array}{l}\text { Estándar de } \\
\text { certificación }\end{array}$} & \multicolumn{4}{|c|}{ Emisiones de GEl (kg CO $2 \mathrm{e} / \mathrm{t}$ café verde) } \\
\hline & & $\begin{array}{c}\text { Combustibles } \\
\text { fósiles }\end{array}$ & Leña & Electricidad & Total \\
\hline Coopedota & $\mathrm{CO}, \mathrm{RF}$ & 30,4 & 124,6 & 0,9 & 155,9 \\
\hline Coopellanobonito & $\mathrm{CO}, \mathrm{CJ}, \mathrm{UK}$ & 38,2 & 146,9 & 1,9 & 187,0 \\
\hline Coopronaranjo & $\mathrm{CO}, \mathrm{OR}, \mathrm{UK}, \mathrm{RF}$ & 17,3 & 203,4 & 1,8 & 222,4 \\
\hline Beneficio La Eva & $\mathrm{CO}$ & 48,0 & 306,0 & 1,5 & 355,5 \\
\hline Coopelibertad & $\mathrm{CO}, \mathrm{UK}, \mathrm{RF}$ & 22,8 & 356,7 & 1,7 & 381,2 \\
\hline APOT & OR & 17,7 & 499,9 & 1,1 & 518,7 \\
\hline $\begin{array}{l}\text { Beneficio Santa } \\
\text { Rosa }\end{array}$ & $\mathrm{CO}$ & 113,5 & 451,9 & 2,0 & 567,4 \\
\hline Cafetalera Aquiares & RF & 28,2 & 622,6 & 2.8 & 653,6 \\
\hline Promedio & & 39,5 & 339,0 & 1,7 & 380,2 \\
\hline
\end{tabular}

CO: Producción convencional; RF: Rainforest; CJ: Comercio Justo; UK: UTZ Kapeh; OR: producción orgánica; APOT: Asociación de Productores Orgánicos de Turrialba.

La emisión de GEl por el uso de la energía eléctrica en Costa Rica es insignificante, porque el $82 \%$ de ésta se 
genera en centrales hidroeléctricas (MIDEPLAN, 2008), que se considera como una energía limpia que no emite GEl en el largo plazo (Chacón, Montenegro \& Sasa, 2009).

\section{Huella de carbono en la cadena de producción de café en Costa Rica}

La cadena productiva del café en Costa Rica presentó una huella de carbono con una fijación neta de entre 2,4 y 13,0 $\mathrm{kg} \mathrm{CO}$ e/kg de grano de café verde (Cuadro 7). Esto significa que, en promedio, cada $\mathrm{kg}$ de grano de café verde producido secuestra $7,6 \mathrm{~kg} \mathrm{CO} \mathrm{CO}_{2}\left(8,1 \mathrm{t} \mathrm{CO}_{2} \mathrm{e} / \mathrm{ha} / \mathrm{año}\right)$. Se encontró una tendencia que indica que la huella de carbono en la cadena de producción de café no fue afectada por los sistemas de certificación. Sin embargo, se hallaron dos grupos de estándares de certificación, de acuerdo a su capacidad de secuestrar GEI. El primer grupo, con la mayor remoción de GEl, está compuesto por la producción convencional y la orgánica $\left(13,0\right.$ y $11,4 \mathrm{~kg} \mathrm{CO}_{2} \mathrm{e} / \mathrm{kg}$ de grano de café verde, respectivamente); mientras que en el segundo grupo, con menor fijación neta de carbono, se encuentran los estándares Rainforest, Comercio Justo y UTZ Kapeh $(2,4 ; 5,4$ y $5,6 \mathrm{~kg} \mathrm{CO} 2 \mathrm{e} / \mathrm{kg}$ de grano de café verde, respectivamente; Cuadro 5). Los hallazgos de este estudio resultaron muy superiores a lo encontrado por Noponen, et al. (2012) en Costa Rica y Nicaragua, quienes encontraron una huella de carbono de 0,26-0,67 y 0,12$0,52 \mathrm{~kg} \mathrm{CO}_{2} \mathrm{e} / \mathrm{kg}$ de café verde, para sistemas de producción convencionales y orgánicos, respectivamente.

La leña tiene un impacto negativo en la huella de carbono, ya que se considera una emisión neta en la cadena de suministro del café, a pesar que el carbono atmosférico se ha fijado previamente en esta fuente energética. Las distancias entre granjas y plantas de procesamiento y el tipo de vehículo para transportar el grano de café fueron también claves para la huella de carbono. Los beneficiaderos de café recogieron el café en cereza de todas las fincas evaluadas, que en promedio estaban a 3,5 $\mathrm{km}$ de distancia.

Estos resultados demuestran que los SAF con café, sin certificación o bajo cualquier estándar, son amigables con el medio ambiente, en términos de mitigación del cambio climático, ya que tienen una huella de carbono positiva, es decir, fijan netamente carbono atmosférico. Estos resultados tienen grandes implicaciones, pues confirman el papel de dichos sistemas de producción en la mitigación del cambio climático y su potencial uso en programas de pagos por servicios ambientales, como una opción para el mejoramiento de los medios de vida de las poblaciones rurales. Igualmente, da pautas a los tomadores de políticas 
nacionales de Costa Rica para promover sistemas que ayudan a alcanzar la meta del país de convertirse en carbono neutro para el 2021.

Cuadro 7. Huella de carbon en la ca dena de producción de caré bajo diferentes estándares de certícación en Costa Rica,

\begin{tabular}{|c|c|c|c|c|c|c|}
\hline \multirow[b]{2}{*}{$\begin{array}{l}\text { Esquema de } \\
\text { certificación }\end{array}$} & \multirow{2}{*}{$\begin{array}{l}\text { Produccion } \\
\text { de grano } \\
\text { verde } \\
\text { (kg/halaño) }\end{array}$} & \multicolumn{3}{|c|}{ Flujos de GEl (kg CO${ }_{2}$ eikg cafe verde)* } & \multicolumn{2}{|c|}{ Huella de carbono } \\
\hline & & $\begin{array}{l}\text { Fijación de } \\
\text { carbono }\end{array}$ & $\begin{array}{l}\text { Manejo de } \\
\text { plantaciones }\end{array}$ & Procesamiento & $\mathrm{kg} \mathrm{CO} z e / \mathrm{kg}$ café & tCO $\mathrm{CO}_{2}$ /ha/año \\
\hline Rainforest & 1584 & 3,7 & $=0,841$ & $-0,438$ & 2,4 & 4,0 \\
\hline $\begin{array}{l}\text { Producción } \\
\text { Orgänica }\end{array}$ & 463 & 34.5 & $-2,266$ & -0.437 & 11,4 & 5,3 \\
\hline $\begin{array}{l}\text { UTZ Kapeh } \\
\text { Comercio Justo }\end{array}$ & $\begin{array}{l}1204 \\
1840\end{array}$ & $\begin{array}{l}6,7 \\
6,4\end{array}$ & $\begin{array}{l}-0,730 \\
-0,816\end{array}$ & $\begin{array}{l}-0,381 \\
-0,187\end{array}$ & $\begin{array}{l}5,6 \\
5,4\end{array}$ & $\begin{array}{l}6.7 \\
9,9\end{array}$ \\
\hline $\begin{array}{l}\text { Producción } \\
\text { convencional }\end{array}$ & 1116 & 14,0 & $-0,661$ & $-0,362$ & 13,0 & 14,4 \\
\hline Promedio & 1241 & 13,1 & $-1,063$ & $-0,361$ & 7,6 & 8,1 \\
\hline
\end{tabular}

\section{CONCLUSIONES Y RECOMENDACIONES}

El componente arbóreo presentó una mayor influencia en la fijación de carbono que el esquema de certificación y los cafetos en la fijación y huella de carbono. Así, la producción convencional de café, la cual tiene árboles de $C$. alliodora, presentó la mayor tasa de fijación de carbono (13,9 t $\mathrm{CO}_{2} \mathrm{e} / \mathrm{ha} / \mathrm{año}$ ). La emisión de GEl fue evidentemente superior en las actividades de manejo de las plantaciones de café que en el procesamiento del grano $(5,1 \mathrm{vs} 0,4 \mathrm{~kg}$ $\mathrm{CO}_{2} \mathrm{e} / \mathrm{kg}$ café verde). En manejo de las plantaciones, la principal actividad de emisión de GEl es la fertilización nitrogenada, por lo cual se deben buscar opciones que reduzcan la aplicación de abonos orgánicos e inorgánicos que suministren tal nutriente.

La cadena productiva de café en Costa Rica es amigable con el medio ambiente, en términos de mitigación del cambio climático, dado que fija en promedio $7,6 \mathrm{~kg} \mathrm{CO} \mathrm{CO}_{2} \mathrm{ekg}$ de café verde $\left(8,1 \mathrm{t} \mathrm{CO}_{2} \mathrm{e} / \mathrm{ha} / \mathrm{año}\right)$. No se encontró un impacto del estándar de certificación en la huella de carbono, pero si en el componente arbóreo, pues cada esquema de certificación tienen sus propias normas en cuanto a este tipo de vegetación en las plantaciones de café.

Idealmente, la huella de carbono debe ser estimada para todo el ciclo de plantación, por lo cual se recomienda estimar las emisiones de GEI en el proceso de establecimiento. Adicionalmente, se requiere intensificar el muestreo de plantaciones con diferentes esquemas de certificación para tener datos más precisos, con mayor rigor estadístico y eliminar aquellas fincas con manejo atípico. 
Se recomienda realizar estudios detallados para encontrar factores de emisión propios para las fuentes de GEI en la zona andina, y principalmente en el área cafetera. Es de vital importancia trabajar en las emisiones de fertilizantes nitrogenados orgánicos.

\section{AGRADECIMIENTOS}

Este artículo fue preparado con base en productos del Proyecto del Comité de Evaluación de Sostenibilidad (COSA -Committee on Sustainability Assessment) como una contribución al desarrollo de la metodología COSA. Ver: http://www.sustainablecommodities.org/node/18".

\section{BIBLIOGRAFÍA}

- Albrecht, A. \& Kandji S. T. (2003). Carbon sequestration in tropical agroforestry systems. Agriculture, Ecosystems and Environment. 99: 15-27.

- Amézquita, M. C.; Ibrahim, M.; Llanderal, T.; Buurman, P, \& Amézquita, E. (2005). Carbon sequestration in pastures, silvo-pastoral systems and forests in four regions of the Latin American tropics. Journal of Sustainable Forestry. 25 (1): 31-49.

- Andrade, H. J.; Brook, R. \& Ibrahim, M. (2008). Growth, production and carbon sequestration of silvo-pastoral systems with native timber species in the dry lowlands of Costa Rica. Plant and Soil. 308: 11-22.

- Andrade, H. J.; Segura, M. \& Somarriba, E. En preparación. Ecuaciones de biomasa para estimar biomasa arriba del suelo de componentes leñosos en sistemas agroforestales indígenas.

- Andrade, H. J.; Marín, L. M.; Pachón, D. P. \& Segura, M. A. En edición. Fijación de carbono en sistemas de producción de café (Coffea arabica L.) en el Líbano, Tolima, Colombia.

- Aristizábal, J. \& Guerra, A. (2002). Estimación de la tasa de fijación de carbono en el sistema agroforestal nogal cafetero Cordia alliodora -cacao Theobroma cacao L-plátano Musa paradisiaca. (Tesis de Ingeniería Forestal no publicada). Universidad Distrital, Bogotá, Colombia.

- Ávila, G. (2000). Fijación y almacenamiento de carbono en sistemas de café bajo sombra, café a pleno sol, sistemas silvo-pastoriles y pasturas a pleno sol. (Tesis de maestría no publicada). CATIE, Turrialba, Costa Rica.

- Ávila, G.; Jiménez, F.; Beer, J.; Gómez, M. \& Ibrahim, M. (2001). Almacenamiento, fijación de carbono y valoración de 
servicios ambientales en sistemas agroforestales. Agroforestería en las Américas, 8(30): 32-35.

- Avilés, I. I. (2009). Fijación biológica de nitrógeno y almacenamiento de carbono en agrosistemas de producción de café (Coffea arabica L) en Puerto Rico. (Tesis de maestría no publicada). Universidad de Puerto Rico, Mayagüez, Puerto Rico.

- Beer, J.; Harvey, C.; Ibrahim, M.; Harmand, J. M.; Somarriba, E. \& Jiménez, F. (2003). Servicios ambientales de los sistemas agroforestales. Agroforestería en las Américas, 10: 80-87.

- Cairns, M. A.; Brown, S.; Helmer, E. H. \& Baumgardner, G.A. (1997). Root biomass allocation in the world'supland forests. Oecologia, 111: 1-11.

- Centro Agronómico Tropical de Investigación y Enseñanza (CATIE). (1994). Deglupta (Eucalyptus deglupta), especie de árbol de uso múltiple en América Central. Serie Técnica, Informe Técnico No. 240. Turrialba, Costa Rica: Autor.

- Chacón, A. R.; Montenegro, J. \& Sasa, J. (2009). Inventario nacional de emisión de gases con efecto invernadero y de absorción de carbono en Costa Rica en el 2000 y 2005. San José, Costa Rica: Instituto Meteorológico Nacional.

- Chaves, E. \& Fonseca, W. (1991). Ciprés, Cupressus lusitanica Miller, especie de árbol de uso múltiple en América Central. Serie Técnica, Informe Técnico No. 168. Turrialba, Costa Rica: CATIE.

- Environment Canada (EC). (2007). National Inventory Report 1990-2005: gas sources and sinks in Canada. Gatineau, Quebec: Autor.

- Giovannucci, D. \& Koekoek, F. J. (2003). The state of sustainable coffee: a study of twelve major markets. California, USA: Feriva.

- Gonzalez, D. (2001). Estudio de casos sobre combustibles forestales, Panamá. Proyecto Información y análisis para el manejo forestal sostenible: integrando esfuerzos nacionales e internacionales en 13 países tropicales en América Latina. Buenos Aires, Argentina: Unión Europea-FAO.

- Hergoualc'h, K.; Blanchart, E.; Skiba, U.; Henault, C. \& Harmand, J. M. (2012). Changes in carbon stock and greenhouse gas balance in a coffee (Coffea arabica) monoculture versus an agroforestry system with Inga densiflora, in Costa Rica. Agriculture, Ecosystems and Environment, 148: 102-110.

- Intergovernmental Panel on Climate Change (IPCC). (2003). National Greenhouse Gas Inventories Programme Intergovernmental. Good Practice Guidance for Land Use, Land-Use Change and Forestry. En IPCC Good Practice Guidance for LULUCF, Chapter 4: Supplementary Methods and Good Practice Guidance Arising from the Kyoto Protocol, Panel on Climate Change (pp. 113-116). Hayama, Kanagawa, Japón: Autor. 
- Intergovernmental Panel on Climate Change. (IPCC) (2005). Métodos complementarios y orientación sobre las buenas prácticas que emanan del Protocolo de Kyoto. Orientación del IPCC sobre las buenas prácticas para uso de la tierra, cambio de uso de la tierra y silvicultura. Capítulo 4. Obtenido desde: http://www.ipccnggip.iges.or.jp/public/gpglulucf/gpglulucf.html.

- Intergovernmental Panel on Climate Change (IPCC) (2006). IPCC Guidelines for National Greenhouse Gas Inventories, Intergovernmental Panel on Climate Change National Greenhouse Gas Inventories Programme. Obtenido desde: http://www.ipcc-nggip.iges.or.jp/public/2006gl/.

- Intergovernmental Panel on Climate Change (IPCC). (2007). IPCC Fourth Assessment Report: Climate Change 2007. Obtenido desde: http://www.ipcc.ch/publications_and_data/ar4/syr/en/figure -spm-3.html.

- Johnson, J.; Fransluebbers, A. J.; Weyers, S. L. \& Reicosky, D. C. (2007). Agricultural opportunities to mitigate greenhouse gas emissions. Environmental pollution, 150: 107-124.

- Ministerio de Planificación Nacional y Política Económica (MIDEPLAN). (2008). Sistema de Indicadores sobre Desarrollo Sostenible (SIDES). Generación eléctrica de Costa Rica por tipo de generación (en GigaWatt-hora): 1990-2000. Obtenido desde: http://www.mideplan.go.cr/sides/ambiental/29-7.htm.

- Montagnini, F; Nair PKR. (2004). Carbon sequestration: an underexploited environmental benefit of agroforestry systems. Agroforestry Systems. 61-62(1-3): 281-295.

- Noponen, R.; Edwards-Jones, G.; Haggar, J. P.; Soto, G.; Attarzadeh, N. \& Healey, J. R. (2012).Greenhouse gas emissions in coffee grown with differing input levels under conventional and organic management. Agriculture, Ecosystems and Environment, 151, 6- 15.

- Oelbermann, M.; Voroney, R. P. \& Gordon, A. M. (2004). Carbon sequestration in tropical and temperate agroforestry systems: a review with examples from Costa Rica and Southern Canada. Agriculture Ecosystems and Environment. 104, 359-377.

- Philpott,S.; Bichier, P.; Rice, R. \& Greenberg, R. (2007). Field-Testing Ecological and Economic Benefits of Coffee Certification Programs. Conservation Biology, 21(4): 975985.

- Segura, M.; Kanninen, M. \& Suárez, D. (2006). Allometric models for estimating aboveground biomass of shade trees and coffee bushes grown together. Agroforestry Systems. 68(2): 143-150.

- Soto-Pinto, L.; Anzueto, M.; Mendoza, J.; Jiménez-Ferrer, G. \& De Jong, B. (2010). Carbon sequestration through agroforestry in indigenous communities of Chiapas, Mexico. Agroforestry Systems. 78: 39-51. 
- United States Agency for International Development (USAID) (2006). Central America and Dominican Republic quality coffee program (CADR QCP). Final Report. 49 p. Washington, D.C.: Author.

- World Resource Institute (WRI) \& World Business Council for Sustainable Development (WBCSD). (2007). Guidelines for quantifying GHG reductions from grid-connected electricity projects. Ginebra: Author.

- Wunder, S. (2007).The efficiency of payments for environmental services in tropical conservation. Conservation Biology. 21(1): 48-58.

1. Ing. Forestal; M.Sc. en Socioeconomía Ambiental. DocenteInvestigadora, Facultad de Ingeniería Forestal, Universidad del Tolima, Email: masegura@ut.edu.co, Grupo de Investigación Producción Ecoamigable de Cultivos Tropicales (PROECUT).

2. Ing. Agrónomo; M.Sc. en Agroforestería Tropical; PhD en Agroforestería Tropical. Docente-Investigador, Facultad de Ingeniería Agronómica, Universidad del Tolima, Email: hjandrade@ut.edu.co, Grupo de Investigación Producción Ecoamigable de Cultivos Tropicales (PROECUT). 